Journal of Computer Science 5 (12): 1082-1090, 2009

ISSN 1549-3636

(C) 2009 Science Publications

\title{
Energy Efficient Hidden Markov Model Based Target Tracking Mechanism in Wireless Sensor Networks
}

\author{
${ }^{1} \mathrm{~B}$. Amutha and ${ }^{2} \mathrm{M}$. Ponnavaikko \\ ${ }^{1}$ Department of Computer Science and Engineering, \\ Sri Ramaswamy Memorial University, Kattankulathur, Chennai 603 203, India \\ ${ }^{2}$ Bharathidasan University, Trichy, India
}

\begin{abstract}
Problem statement: Target tracking is a challenging application in Wireless Sensor Networks (WSNs) because it is computation-intensive and requires real-time location processing. This study proposes a practical target tracking system based on the Hidden Markov Model in a distributed signal processing framework. In this framework, wireless sensor nodes perform target detection and tracking, whereas target localization requires the collaborative signal processing between wireless sensor nodes for improving the location accuracy and robustness. Approach: For carrying out target tracking under the constraints imposed by the limited transmission capabilities of the wireless sensor nodes, the HMM model and the particle filter approach are adopted in single wireless sensor node due to their outstanding performance and light computational calculations. Furthermore, a progressive multi sensor localization algorithm is proposed in distributed wireless sensor network considering the tradeoff between the localization accuracy of the target and the resource constraints of sensor nodes. Results: Finally, a real world target tracking experiment had been illustrated for static and mobile targets. Here blind child is considered as the target to be tracked within the sensor network. Conclusion: Mathematical analysis and the real world results showed that the target tracking system based on a distributed WSN make efficient use of the communication resources and achieve accurate target tracking successfully.
\end{abstract}

Key words: Hidden Markov model, particle filter, Gaussian noise, TDOA, entropy

\section{INTRODUCTION}

Wireless Sensor Networks (WSN) have become one of the most promising and interesting areas over the past few years. These networks are very large systems comprised of small sized, low power, low-cost sensor devices which collect information about the physical environment. The flexibility, self-organization, fault tolerance, high sensing fidelity, low-cost and rapid deployment characteristics of sensor networks have created many new and exciting application areas for remote sensing. The purpose of target tracking algorithm is to determine the target's trajectories using sensor measurements. An additional prior information is the dynamic nature of targets.

A more general tracking scenario consists of multiple sensors may have different precisions and accuracy and can produce different kinds of measurements. Due to the low energy constraints in WSN, the network is divided into clusters, each of which will have a cluster-head, which is equipped with a GPS device. This minimizes the energy consumption by avoiding all the nodes needing to send data to a distant base station, which is away from the vicinity of the sensor nodes in the target tracking scenario.

The Cluster Head know about all sensors belonging to its cluster. The informations are: (1) sensor identity, (2) location and (3) energy level (4) the density of the sensor nodes in the cluster. When tracking a moving target, \{say human\} and to receive the target information from the very nearer sensor nodes, the cluster head based on the current information is used for collecting the information about the target. The objective is to provide automatic detection based on Hidden Markov Model (HMM) and tracking algorithm based on Modified Particle Filter (MPF) in order to operate in a densely cluttered environment in an energy efficient way ${ }^{[1]}$.

Related works: A target detection method is presented based on the Hidden Markov Model (HMM). The scattering of information from multiple targets is

Correspondign Auhtor: B. Amutha, Department of Computer Science and Engineering, Sri Ramaswamy Memorial University, Kattankulathur,Chennai 603 203, India 
modeled as an HMM with the number of underlying states have been treated as infinite, from which a full posterior distribution on the number of states associated with the targets is inferred and the target-dependent states have been identified. Anomaly detection techniques have been devised to address the limitations of misuse detection approaches for intrusion detection with the model of normal behaviors. A hidden Markov Model is a useful tool to model sequence information, an optimal modeling technique to minimize falsepositive error while maximizing detection rate. In spite of high performance, however, it requires large amounts of time to model normal behaviors and determines intrusions, making it difficult to detect intrusions in real-time ${ }^{[2]}$.

With sufficiently large numbers of training data, it might show better performance and reduction of computational costs. This method opened a new way of utilizing computation intensive anomaly detection technique in the real world, based on behavioral constraints imposed by security policies and on models of typical behavior for users. For the deployment of the system in the real world, an automatic mechanism to adjust the threshold for HMM appropriately is needed.

Target tracking: Particle Filtering $(\mathrm{PF})$ has been widely used in solving nonlinear/non Gaussian filtering problems. Inferring to the target tracking in a Wireless Sensor Network, Distributed PF was used due to the limitation of nodes computing capacity. Synchronous DPF and GMM parameters transferred DPF have their own disadvantages which limit their using range. In this study, a novel filtering method-asynchronous DPF (ADPF) for target tracking in WSN is proposed. With incremental updating GMM parameters, ADPF can use the sampling information effectively. And ADPF can also deal with the situation of different number of nodes in different cluster when using the dynamic cluster structure. Simulation result shows that ADPF has better performance than other two typical DPF algorithms.

Sensor systems are not always equipped with the ability to track targets. Sudden maneuvers of a target can have a great impact on the sensor system, which will increase the miss rate and rate of false target detection.

The use of the generic Particle Filter (PF) algorithm is well known for target tracking, but it can not overcome the degeneracy of particles and accumulation of estimation errors. This algorithm uses the Radial-Basis Function Network (RBFN) in the sampling step for dynamically constructing the process model from observations and updating the value of each particle.
With the RBFN sampling step, PF-RBF can give an accurate proposal distribution and maintain the convergence of a sensor system. Simulation results verify that PF-RBF performs better than the Unscented Kalman Filter (UKF), PF and Unscented Particle Filter (UPF) in both robustness and accuracy whether the observation model used for the sensor system is linear or nonlinear. Moreover, the intrinsic property of PFRBF determines that, when the particle number exceeds a certain amount, the execution time of PF-RBF is less than UPF. This makes PF-RBF a better candidate for the sensor systems which need many particles for target tracking $^{[1-4]}$.

Tracking a target in sensor networks is quite challenging regardless of the energy consumption due to resource-constrained of the network devices. In a tracking task the sensor management addresses the problem of choosing informative sensors needed to obtain information about the target state and therefore maximize the network lifetime for a given cost of communication and computation. The problem here is to selecting the best nodes for tracking a target in a distributed wireless sensor network.

Energy efficiency: This study revises and evaluates an energy-efficient distributed collaborative signal and information processing framework for acoustic target tracking in wireless sensor networks. The distributed processing algorithm is based on mobile agent computing paradigm and sequential Bayesian estimation. At each time step, the short detection reports of cluster members will be collected by cluster head and a sensor node with the highest Signal-ToNoise Ratio (SNR) is chosen there as reference node for Time Difference Of Arrive (TDOA) calculation.

To track mobile nodes two particle filters, bootstrap and unscented particle filter, which estimate the actual position and predict future locations, are used. The particle filters have been widely used in tracking algorithms, but their energy efficiency has received less attention. The approach has been implemented with PF and UPF for nonlinear Gaussian problems. Finally, in this study we assumed an intercluster communication and limited ourselves to consider a single cluster in the evaluation of the energy consumption. Current study is investigating the implementation of algorithms to report tracking samples to multiple cluster heads ${ }^{[5-7]}$.

\section{MATERIALS AND METHODS}

Objectives of the model: The proposed system utilize new tracking algorithm which combines HMM and 
modified Particle Filter for dynamically moving object. Target Detection done with Hidden Markov Models (HMM) Produces the maximum likelihood estimates via the real time viterbi optimization algorithm. HMM is used to detect the future path of the blind human. Target Tracking done with Modified Particle Filter (MPF) improves accuracy with the help of the Entropy method.

This Proposed system tracks, detects and locates targets in a timely and energy efficient manner. Evaluates an energy-efficient approach that consumes less energy while achieves optimal accuracy for Target tracking in wireless sensor networks. In the earlier cases, the cluster structure is fixed. In the proposed system, dynamic cluster structure is used to track the target and the target's future direction is decided according to the obstacles identified in the forwarding path.

Optimization of utility function: Target tracking is usually a challenging application for wireless sensor networks (WSNs) because it is always computationintensive and requires real-time processing. This study proposes a practical target tracking system based on the hidden Markov model with modified particle filtering in a distributed signal processing framework. In the proposed framework, wireless sensor nodes perform target detection, classification and tracking, whereas target localization requires the collaboration between wireless sensor nodes for improving the accuracy and robustness. For carrying out target tracking under the constraints imposed by the limited capabilities of the wireless sensor nodes, some practically feasible algorithms, such as the HMM model and the Particle Filter approach, are adopted in single wireless sensor nodes due to their outstanding performance and light computational burden.

Furthermore, a progressive secured multi-view localization algorithm is proposed in distributed wireless sensor network considering the tradeoff between the accuracy and energy consumption. Finally, a real world target tracking experiment will be illustrated for static and mobile targets. Proposed Results from theoretical analysis showed that the proposed target tracking system based on a distributed WSN can make efficient use of scarce energy and communication resources and achieve target tracking successfully.

The main aim of the work is to detect and locate the target within the specified WSN framework. The target within a real time environment is considered here, the dynamic nature of the target is chosen for the project. As there are no sufficient tracking models available for target tracking in an accurate manner, the capabilities of the Hidden Markov Model combined with Particle Filter is considered to form a new tracking algorithm for dynamically moving object.

The entire state space of the object movement area is divided into subspaces and the state sequence corresponds to the normal movement step of the object. As the starting point and the direction are known, it is possible to detect the future path of the object without any lag according to HMM. In certain cases the direction may also be unknown due to some obstacles or due some uncertainties in the path. Those cases have been taken into consideration and a modified particle filter algorithm along with HMM is an efficient choice for providing the exact location of the dynamically moving target. In this study, a blind child is considered as the moving target.

Initial summary: A Gps Equipped base station must be constructed at every school. The antenna can cover a distance of 5-10 km radius. The School must have an internet enabled kiosk centre, where the information about the children are to be uploaded in the internet kiosk (through a service provider-here the School itself).

Gps Transceiver can transmit the signal to all the wireless sensors connected in the predator device (Future path finding device), which is constructed as a model of a school belt, that every blind child can wear. The look up table of data for each and every blind child's home location data is inputted in to the school server. For this purpose, we made an initial study of the home locations of the children. Gps transceiver is taken in hand and the estimated GPS Location coordinates of each and every children of the school has been updated in the web server, which procure the locations of all student homes and their Geolocation points are mentioned in the School database along with the children details.

The system we propose possess following features: The entire child localization, tracking and navigation problem consists of five modules:

- Obstacle detection using sonar

- Detection of hanging obstacles

- Path guidance using GPS

- Provision of remote assistance in case of an circumstantial hazard

- Auditory and vibratory alert signals through headphones

The safe path finding mechanism involves the entire trajectory of the child's movements on earth, 
when the child is in an outdoor environment. As the roaming area is specified, then the entire area map is downloaded on to the mobile phone of the caretaker of the child. A set of specified paths in which the child's expected roaming area is taken as $\mathrm{Si}$ where $\mathrm{i}$ range from 1 to $n$ :

$\mathrm{Si}=\mathrm{I}(1,2,3 \ldots \ldots \mathrm{n})$

The child's walking style varies with height and mobility:

$\mathrm{Mh}=$ Height of children

$\mathrm{Mm}=$ Mobility of $\mathrm{M}$ children

indicates the walking speed of the children:

- As height varies with respect to age, age is considered here as an indirect measure of mobility. Mobility is considered as the stepwise movement of the child. It is observed that, the distance covered by a normal child in one step can be covered by the blind child of same height and weight in two steps:

- So blind people model take two steps to cover the same distance

- But another ambiguity is blind people take first step, and then the second step comes to same position of first step

So it will take more time for the blind child to cover the same distance, as a normal child of the same age (height, weight) covers. Suppose a normal person take $1 \mathrm{sec}$ for 1 step (observed), then a blind person will take $2.7 \mathrm{sec}$ for one step:

$\mathrm{Mh} * \mathrm{Mm}=$ Walking style

The stepwise movement of $\mathrm{M}$ children is analyzed based on the above matrix. An average stepping algorithm is introduced in to the GPS receiver, and the instructions are given to the child for making the next movement.

Using Maximum Likelihood (ML) solution:

$\hat{\mathrm{H}}=\operatorname{argmax} \mathrm{f}(\mathrm{r} / \mathrm{Hn})$, Where $\mathrm{n}=1,2,3, \ldots \ldots \ldots$

$\mathrm{Hn}=$ Minimum number of hops $=\mathrm{n}$

From source to specific node

[Blind School to a particular student] at Euclidean distance $r$.

$\mathrm{f}(\mathrm{r} / \mathrm{Hn})=2 \mathrm{D}$ Poisson distribution

$\mathrm{r}<\mathrm{R}$ implies $\mathrm{H}$
That is specific node is within one hop distance from the source.

Mobile target (blind child) tracking algorithm: Let there may be $\mathrm{n}$ number of device readers (We assumed only one GPS Transceiver to track the device for simplicity) u number of tracking objects (blind children) and $\mathrm{m}$ number of reference nodes, with known location coordinates. Let SS be the sample set that is recorded at regular intervals. The sample set contains the following tuples:

$\mathrm{SS}^{\mathrm{t}}=\left\{\mathrm{S}_{\mathrm{i}}, \theta_{\mathrm{j}}, \mathrm{i}=1,2 \ldots . \mathrm{u}, \mathrm{j}=1,2, \ldots \mathrm{m}\right\}$

where, $\mathrm{t}$ is the time interval.

$\mathrm{S}_{\mathrm{i}}=\left\{\mathrm{S}_{1}, \mathrm{~S}_{2}, \ldots \ldots, \mathrm{S}_{\mathrm{n}}\right\}$

$\Theta_{\mathrm{j}}=\left\{\Theta_{1}, \Theta_{2}, \ldots \ldots, \Theta_{\mathrm{n}}\right\}$

Here $S_{i}$ is the set containing the signal strength of $\mathrm{i}^{\text {th }}$ tracking object from $\mathrm{n}$ readers and $\Theta_{\mathrm{j}}$ is the set containing the signal strength of $\mathrm{j}^{\text {th }}$ reference object from $\mathrm{n}$ readers. The algorithm is explained in the following steps:

Step 1: Compute the sample set $S S^{t}$ at regular intervals Let the sample set be:

$\mathrm{SS}_{\mathrm{k}}^{\mathrm{t}}=\left\{\theta_{\mathrm{j}}, \mathrm{S}_{\mathrm{i}}, \mathrm{j}=1,2, \ldots \mathrm{m}, \mathrm{i}=1,2, \ldots \mathrm{u}\right\}$

at time interval $\mathrm{t}$

Step 2: If the time interval to find the location of the tracking object is not with in the range i.e., not at regular intervals than go to step 4 for prediction.

Step 3: If the time interval to find the location of the tracking object is with in the range i.e., at regular intervals then finalize the sample set $\hat{\mathrm{S}} \hat{S}^{t}$ by capturing the signal strength of tracking object and its corresponding reference nodes through $n$ readers i.e.:

$\hat{\mathrm{S}} \hat{\mathrm{S}}^{\mathrm{t}}=\left\{\mathrm{S}_{\mathrm{i}}, \Theta_{\mathrm{j}}\right\}$

Go to step 5

Step 4: The measurement history contains the record of sample sets of last ten intervals. Let the final set be:

$$
\hat{\mathrm{S}} \hat{\mathrm{S}}^{\mathrm{t}}=\left\{\mathrm{S}_{\mathrm{i}}, \Theta_{\mathrm{j}}\right\}
$$

Step 5: Calculate the Euclidean distance between the signal strength of tracking object and reference nodes neared to the tracking object: 


$$
E_{j}=\operatorname{sqrt}\left(\sum_{i=1}^{n}\left(\theta_{i}-S_{i}\right)^{2}\right) \text { where } j=1, \ldots, m
$$

Step 6: After the Euclidean distance is calculated, based on this distance select the set of $\mathrm{k}$ reference tags neared to the tracking object. Let it be:

$$
\mathrm{E}_{\mathrm{q}}=\left\{\mathrm{E}_{1}, \mathrm{E}_{2}, \ldots ., \mathrm{E}_{\mathrm{k}}\right\} \text { where } \mathrm{q}=1, \ldots \mathrm{k}
$$

Step 7: The weighting factors are estimated using the following formula:

$$
w_{j}=\frac{1 / E_{i}^{2}}{\sum_{i=1}^{k} 1 / E_{i}^{2}}
$$

Step 8: The co-ordinates of the tacking object is given by:

$$
(\mathrm{x}, \mathrm{y})=\sum_{\mathrm{l}=1}^{\mathrm{k}} \mathrm{W}_{\mathrm{l}}\left(\mathrm{x}_{1}, \mathrm{y}_{1}\right)
$$

Let $\left\{S_{k}{ }^{(t-1)}, W_{k}^{(t-1)}, K=1, \ldots, M\right\}$ denote the sample set at time $t-1$, where $S_{k}^{(t-1)}$ is the $K^{\text {th }}$ sample for the prior distribution of target state $\mathrm{P}\left(\mathrm{X}^{(\mathrm{t}-1)} \mid \check{\mathrm{Z}}^{(\mathrm{t}-1)}\right)$ and $\mathrm{W}_{\mathrm{k}}^{(\mathrm{t}-1)}$ its probability weight. The $\mathrm{K}^{\text {th }}$ sample of the predicted state at time $\mathrm{t}$ is denoted by $\hat{\mathrm{S}}_{\mathrm{k}}{ }^{(\mathrm{t})}$. The estimation algorithm consists of the following steps:

Step 1: Initialization:

Step 2: Prediction and update:
a. Apply $\mathrm{P}\left(\mathrm{S}^{(t)} \mid \mathrm{S}_{\mathrm{k}}{ }^{(\mathrm{t}-1)}\right)$ to compute each $\hat{\mathrm{S}}_{\mathrm{k}}{ }^{(\mathrm{t})}$ : sample $\hat{S}_{k}{ }^{(t)}$ from $P\left(S^{(t)} \mid S_{k}^{(t-1)}\right)$

b. Evaluate the importance weights:

$$
\hat{\mathrm{W}}_{\mathrm{k}}^{(\mathrm{t})}=\mathrm{P}\left(\mathrm{Z}^{(\mathrm{t})} \mid \hat{\mathrm{S}}_{\mathrm{k}}^{(\mathrm{t})}\right)
$$

c. Normalize the weights:

$$
\mathrm{W}_{\mathrm{k}}^{(\mathrm{t})}=\frac{\hat{\mathrm{W}}_{\mathrm{k}}^{(\mathrm{t})}}{\sum_{\mathrm{k}=1}^{\mathrm{k}} \hat{\mathrm{W}}_{\mathrm{k}}^{(\mathrm{t})}}
$$

Step 3: Resampling:
a. Resample $M$ particles $\mathrm{S}_{\mathrm{k}}{ }^{(\mathrm{t})}$ from $\hat{\mathrm{S}}_{\mathrm{k}}{ }^{(\mathrm{t})}$
b. Set $\mathrm{t}=\mathrm{t}+1$ and go to step 2

The update step scales the weight of each particle according to the likelihood function value at the particle. As the result, some particles become heavier, and some too small to be included in the particle set.
The weight which is considered here is the time taken for the child to take decision to move further in the same path or to choose an alternate path based on the obstacle found after each step.The resampling step draws a set of $\mathrm{M}$ samples with the same weight from the updated particle set, thus keeping the total number of particles to a constant at each iteration. This ensures the amount of computation is constant at each iteration for practical reasons, only if there is no change of direction occurs. By incorporating this particle filter in location estimation algorithm the location of moving child can be tracked.

Dynamic clustering architecture: Each application has a different set of tasks to be carried out; three basic types of tasks exist in a sensor network: Sensing, processing and communication. Sensing is the process of collecting data from the physical world. Data from different sensors are processed inside or outside the network to obtain a better understanding of the environment. Communication enables collaborative signal and data processing from multiple sensors and delivery of results to interested users ${ }^{[5-8]}$.

Target detection: Detection requires that the system discriminate between a target's absence and presence. Successful detection requires a node to correctly estimate a target's presence while avoiding false detections in which no targets are present.

The key performance metrics for detection include the probability of correct detection, or $\mathrm{PD}$, the probability of false alarm, or PFA and the allowable latency, TD between a target's presence and its eventual detection. Hidden Markov Models (HMMs) have been successfully applied to model such sequential data for target detection and classification problems. Each HMM state represents a set of generally contiguous target-sensor orientations over which the signal statistics are relatively invariant. The probability of transitioning from one state to another on consecutive measurements is modeled as a Markov process.

An HMM includes a sequence of observations $\mathrm{o}=\{01 ; \mathrm{o} 2 ;::: ; \mathrm{oT}\}$ and a sequence of hidden underlying states $\mathrm{s}=\{\mathrm{s} 1 ; \mathrm{s} 2 ;::: ; \mathrm{sT}\}$ which follow a Markov process. In many applications, the first order Markov chain is used, i.e., the current state variable only depends on the previous state regardless of the history $\mathrm{P}(\mathrm{st} \mid \mathrm{s} 1$; $\mathrm{s} 2$; : : : ; st-1) $=\mathrm{P}(\mathrm{st} \mid \mathrm{st}-1)$; the observation is then independently generated given the state.

Typically maximum-likelihood HMM parameter learning is performed via the EM algorithm, with the 
number of states assumed known. Assume we use an HMM with $\mathrm{M}$ states and $\mathrm{C}$ possible observations. The parameters of the model are $\left(\pi^{\mathrm{M}}, \mathrm{A}^{\mathrm{M} \times \mathrm{M}}, \mathrm{B}^{\mathrm{M} \times \mathrm{C}}\right)$ with $\pi$ being the initial-state probability, $\mathrm{A}$ the transition matrix of $P\left(s_{t} \mid s_{t-1}\right)$ and $B$ the observation matrix of $\mathrm{P}\left(\mathrm{O}_{\mathrm{t}} \mid \mathrm{S}_{\mathrm{t}}\right)$ :

$$
p\left(o_{t} \mid s_{t-1}=j\right)=\sum_{i=1}^{M} a_{j i} b_{i}\left(o_{t}\right)
$$

The maximum entropy characterization yields a practical optimization algorithm to determine parameters of the model density given moments of the ensemble. A maximum-entropy estimate of the postmeasurement state provides a simplified "mean-field" approximation to the Bayesian update. This estimate is substantially cheaper to calculate than the full Bayesian estimate and may be a practical alternative when computational requirements for the latter exceed available resources. The entropy itself also serves as a useful measure of the information content of the observations and its rate of degradation over time. A broad range of current sensor network applications involve surveillance.

Tracking involves maintaining the target's position as it evolves over time due to its motion in a region covered by the sensor network's field of view. Successful tracking requires that the system estimate a target's initial point of entry and current position with modest accuracy and within the allowable detection latency, TD. Implicit in this requirement is the need for target localization. The tracking performance requirements dictate that tracking accuracy, or the maximum difference between a target's actual and estimated position, be both bounded and specified, within limits, by the user.

The main idea is representing the solution space with a set of parameters and defining a probability distribution on these parameters. Then, two successive steps are iterated-sampling from the existing distribution and updating this distribution using a subset of samples.

Particle filters proceed the computation of posterior distribution through discrete approximations to the exact one. Particles are points chosen from posterior distribution in each time step (Nt particles):

$$
\begin{aligned}
& \hat{p}\left(x_{t} \mid y_{1: t-1}\right)=\sum_{j=1}^{N_{t-1}} p\left(x_{t} \mid x_{t-1}^{(j)}\right) \hat{p}\left(x_{t-1}^{(j)} \mid y_{1: t-1}\right) \\
& \tilde{p}\left(x_{t} \mid y_{1: t}\right)=\tilde{C}_{t}^{-1} p\left(y_{t} \mid x_{t}\right) \hat{p}\left(x_{t} \mid y_{1: t-1}\right) \tilde{C}_{t} \text { (const.) }
\end{aligned}
$$

Particle filter: Suppose the target's present location in $\mathrm{TD}_{\mathrm{i}-1}$ is $\left(\mathrm{x}_{\mathrm{i}-1} ; \mathrm{y}_{\mathrm{i}-1}\right)$ and $\left(\mathrm{x}_{\mathrm{i}} ; \mathrm{y}_{\mathrm{i}}\right)$ in TDi. Then we can estimate the target's speed as:

$$
\mathrm{u}=\frac{\sqrt{\left(\mathrm{x}_{\mathrm{i}}-\mathrm{x}_{\mathrm{i}-1}\right)^{2}+\left(\mathrm{y}_{\mathrm{i}}-\mathrm{y}_{\mathrm{i}-1}\right)^{2}}}{\mathrm{t}_{\mathrm{i}}-\mathrm{t}_{\mathrm{i}-1}}
$$

The direction of the target is given by:

$$
\theta=\cos ^{-1} \frac{\left(x_{i}-x_{i-1}\right)}{\sqrt{\left(x_{i}-x_{i-1}\right)^{2}+\left(y_{i}-y_{i-1}\right)^{2}}}
$$

Based on this information, the predicted location for the target $\left(x_{i+1} ; y_{i+1}\right)$ after a given time $t$ is given by:

$$
\begin{aligned}
& \mathrm{x}_{\mathrm{i}+1}=\mathrm{x}_{\mathrm{i}}+\mathrm{ut} \cos (\theta) \\
& \mathrm{y}_{\mathrm{i}+1}=\mathrm{y}_{\mathrm{i}}+\mathrm{ut} \sin (\theta)
\end{aligned}
$$

To be more precise, it can be shown that the target's next location obeys a two dimensional Gaussian distribution with $\left(\mathrm{x}_{\mathrm{i}+1} ; \mathrm{y}_{\mathrm{i}+1}\right)$ as the mean. In addition to performing the same sensing and calculation tasks as the other member sensors, the cluster head needs to perform the following additional tasks ${ }^{[9-10]}$ :

- To combine the innovations from all tasking sensors and itself into a vector form for estimating the target state using the PF algorithm

- To choose the sampling interval $\Delta \mathrm{ti}$ for step $\mathrm{i}+1$

- To choose tasking sensors $G(i+1)$ for step $i+1$

- To pass the predicted target state $\hat{\mathrm{X}}(\mathrm{i}+1 \mid \mathrm{i})$ and its error covariance $\mathrm{P}(\mathrm{i}+1 \mid \mathrm{i})$ to the cluster $\mathrm{G}(\mathrm{i}+1)$

Energy efficiency: The main concept underlying the proposed energy efficient tracking algorithm is that the $\mathrm{CH}$ should select the active neighbors with the goal of reducing the total energy consumption in the cluster.

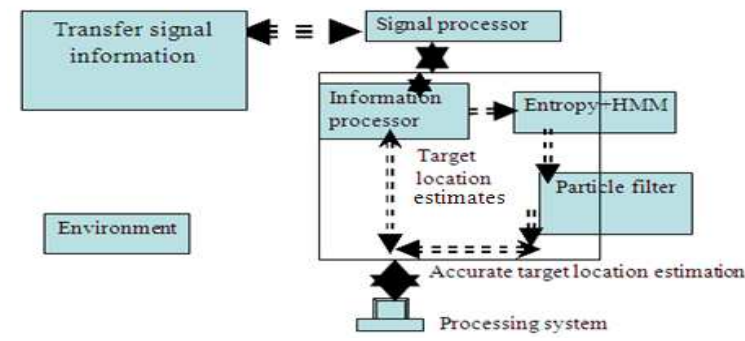

Fig. 1: System architecture 
Figure 1 depicts the system architecture, in which the system is modeled as a target tracking system. Within the wireless sensor network, a blind child is considered as the target. The movement of the child is being tracked by using a 3 axis accelerometer and the particle filter is used for tracking the human with the stepwise movements. As the initial location coordinates have been fed into the information processor and the location estimates are counted by using the accelerometer, the error in estimation is estimated.

Figure 2 depicts the system setup and the noise parameter which is incorporated into the information processor, which reduces the accuracy. From the actual measurements taken, the normalization values have been updated.

For each time step, energy consumption happens mainly in the following operations:

- $\quad$ sensing by tasking sensors

- transmitting/receiving measurement data from tasking sensors to the cluster head

- Broadcasting/receiving by the current cluster head and tasking sensors in the next cluster

The energy level of the sensor nodes according to the distance of the sensor node from the cluster head. It means that if the destination or the base station from where the sensor node is located decides the amount of energy used by the sensor nodes. Energy calculation should also be made based on the distance from the cluster head, as it acts as the base station for a particular cluster.

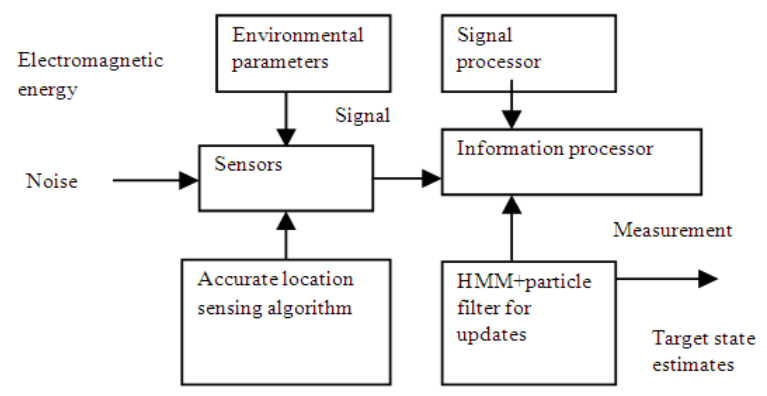

Fig. 2: System setup

Table1: Simulation parameter setup

\begin{tabular}{ll}
\hline Simulator & Ns-2 \\
\hline Examined protocols & DSDV and AODV \\
Simulation duration & $99.68194564 \mathrm{sec}$ \\
Simulation area & $1000 \times 1000 \mathrm{~m}$ \\
Movement model & straight \\
Maximum speed & $10 \mathrm{~m} \mathrm{sec}^{-1}$ \\
Average speed & $4.83 \mathrm{~m} \mathrm{sec}^{-1}$ \\
Traffic type & $\mathrm{CBR}(\mathrm{UDP})$ \\
Data payload & $512 \mathrm{bytes}_{\mathrm{p} a c k e t}$ \\
Bandwidth & $1 \mathrm{Mb} \mathrm{sec}-1$ \\
\hline
\end{tabular}

Table 1 depicts the simulation scenario used for validating the algorithm.

\section{RESULTS}

The measurements for the blind children under different age groups have been displayed in the following diagrams from Fig. 3-9. It shows that the average walking step increases according to height.

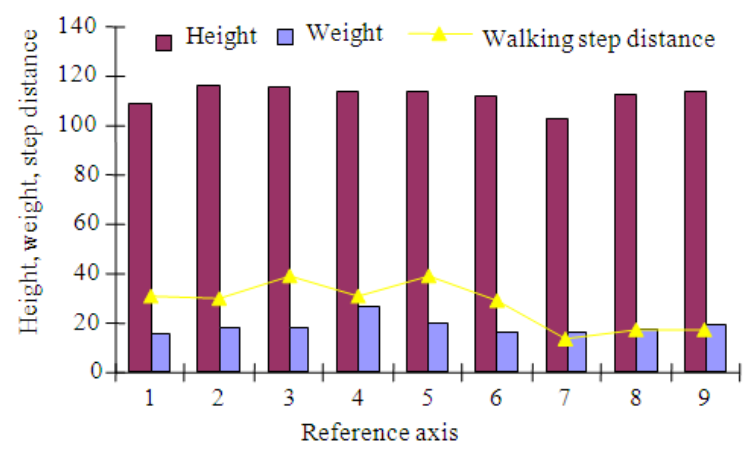

Fig. 3: Walking step variation of blind child STD 1

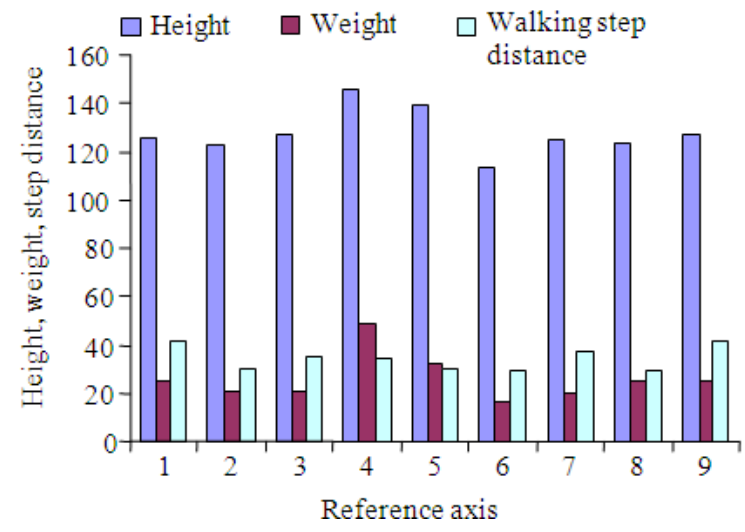

Fig. 4: Walking step variation of blind child STD 1II

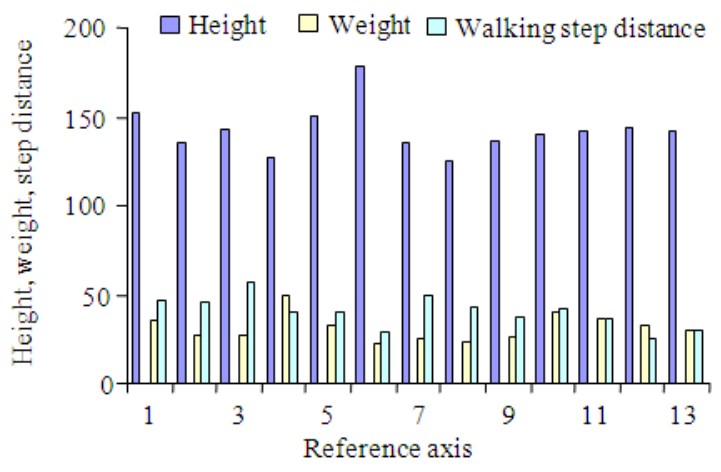

Fig. 5: Walking step variation of blind child STD V 


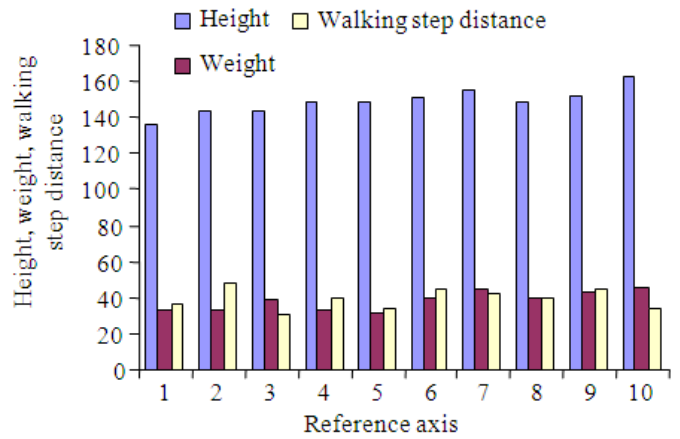

Fig. 6: Walking step variation of blind child STD VIII

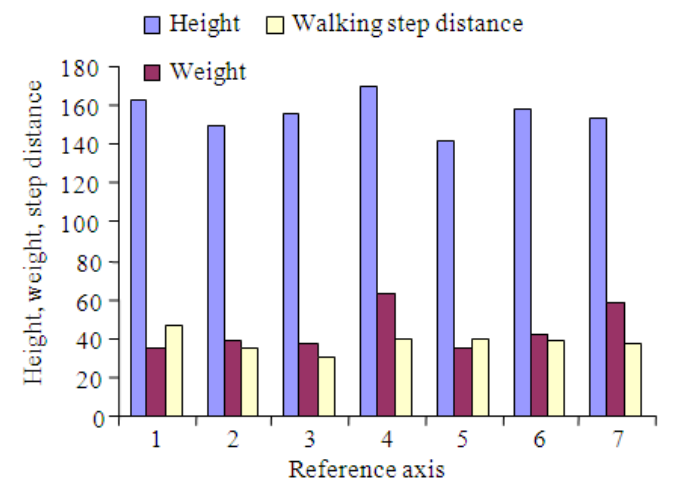

Fig. 7: Walking step variation of blind child STD X

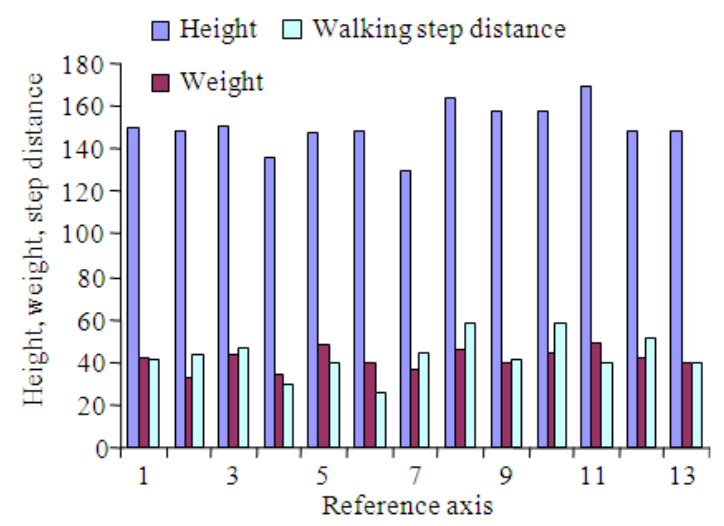

Fig. 8: Walking step variation of blind child STD XII

The average values of height, weight, walking step distance and also the walking speed are used in defining the weighting factor of the algorithm. Also, the latency is proportionate to the time of obstacle detection and the response time of the device. The response time of the device is sampled at regular intervals of 1 second duration. The motion model is considered as straight in the outdoor environment. The cluster head is nothing but the nearest sensor node from the school GPS Transceiver system.

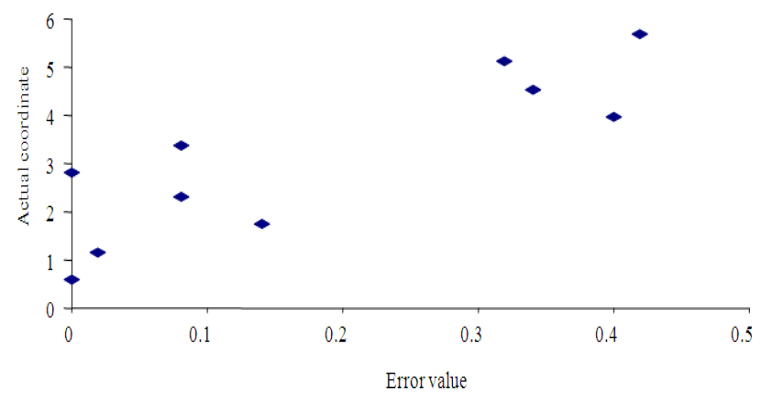

Fig. 9: The error value from the actual location coordinates

The actual location coordinates which varies in terms of step measurement implies the particle filter algorithm updates according to the temporal step differences.

Figure 9 depicts the difference between the accelerometer readings and the particle filter measurements. Particle filter measurements can be smoothened by using a smoothening algorithm by reducing the external noise components is left for future research.

\section{DISCUSSION}

This blind child tracking system worked on tracking the predator device which actually being carried by the blind child. The child can be tracked by using the GPS transceiver located at the school, which covers a maximum distance of 15 kilometers. In the outdoor environment, the blind child without any physical help of others can walk freely from house to school and vice versa, if the child is being tracked. The HMM model along with the particle filter algorithm detect and track the blind child with an accuracy of $85.18 \%$.Out of 27 trials 23 trials were successful with the inclusion of obstacle detection algorithm using sonar sensors for a distance of $1 \mathrm{~km}$.

\section{CONCLUSION}

As it is an efficient target tracking mechanism, only the target localization accuracy and robustness have to be maintained. Also latency avoidance and energy consumption have been incorporated based on the type of obstacles found on the path. Latency is avoided by selecting the nearest possible alternate paths. The real time tracking measurements showed that the algorithm worked successfully with the effective study and real time hardware implementation is on progress. 


\section{REFERENCES}

1. Kreucher, C., D. Blatt, A. Hero and K. Kastella, 2006. Adaptive multi modality sensor scheduling for detection and tracking of smart targets. Digit. Sign. Process., 16: 546-567. DOI: 10.1016/j.dsp.2004.12.008

2. Cho, S.B. and H.J. Park, 2003. Efficient anomaly detection by modeling privilege flows using hidden Markov model. Comput. Secur., 22: 45-55. DOI: 10.1016/s0167-4048[03]00112-3

3. Song, C., H. Zhao and W. Jing, 2009. Asynchronous distributed PF algorithm for WSN target tracking. Proceedings of the 2009 International Conference on Wireless Communications and Mobile Computing: Connecting the World Wirelessly, June 21-24, ACM Press, Leipzig, Germany, pp: 1168-1172. http://portal.acm.org/citation.cfm?id=1582635

4. Wang, X., S. Wang and J.J. Ma, 2007. An improved particle filter for target tracking in sensor systems. Sensors, 7: 144-156. DOI: 10.3390/S7010144

5. Yu, Z., W. Jianming and H. Liu, 2009. An energyefficient target tracking framework in wireless sensor networks. EUROSIP J. Adv. Sign. Process., 2009: 14. DOI: $10.1155 / 2009 / 524145$

6. Yang, H. and B. Sikdar, 2003. A protocol for tracking mobile targets using sensor networks. Proceedings of the 1st IEEE International Workshop on Sensor Network Protocols and Applications, May 11-11, IEEE Xplore Press, Press, USA., pp: 71-81. DOI: 10.1109/SNPA.2003.1203358
7. Reid, D., 1979. An algorithm for tracking multiple targets. IEEE Trans. Automat. Control, 24: 843-854. http://ieeexplore.ieee.org/xpl/freeabs_all.jsp?isNu mber $=24175 \&$ arNumber $=1102177 \&$ isnumber $=241$ $75 \&$ arnumber $=1102177$

8. Chen, W.P. and J.C. Hou, 2004. Dynamic clustering for acoustic target tracking in wireless sensor networks. IEEE Trans. Mobile Comput., 3: 258-271. DOI: 10.1109/TMC.2004.22

9. Wu, H., A. Marshall and W. Yu, 2007. Path planning and following algorithms in an indoor navigation model for visually impaired. Proceedings of the 2nd International Conference on Internet Monitoring and Protection, July 1-5, IEEE Computer Society, Washington DC., USA., pp: 38. http://portal.acm.org/citation.cfm?id=1271138

10. Ashmead, D.H., D. Guth, R.S. Wall, R.G. Long and P.E. Ponchillia, 2005. Street crossing by sighted and blind pedestrians at a modern roundabout. J. Transportat. Eng., 131: 812-821. DOI: 10.1061/(ASCE)0733- 